\title{
Protection Systems against Unmanned Aircraft Vehicle Evaluation
}

\author{
Svajone BEKESIENE
}

\author{
Engineering Managing Department, the General Jonas Žemaitis Military Academy of Lithuania, \\ Silo g. 5A, LT-10322 Vilnius \\ E-mails: Svajone.Bekesiene @lka.lt
}

\begin{abstract}
The purpose of this investigation is to present the protection systems used against the low, slow, and small (LSS) unmanned aerial systems (UAS) capabilities. The results of a market survey are included that highlights potential commercial entities that could contribute some technology that assists in the detection, classification, and neutralization of a LSS UAS. In order to accurately select a protection system which will later be used by all Lithuanian Armed Forces units the nine existing protection systems were analyzed. The analysis was done under the technical specifications for acquisitions and the detailed requirements of the measures were set out on the basis of the Operational Requirements Document.

This article provides the existing protection systems against unmanned aircraft vehicle (PSAUAV) technical conditions analysis with the aim to help to make the choices in defining a protection system that can be adaptive to contemporary and future needs for the Lithuanian Armed Forces. The numerous requirements and expectations for nine PSAUAV were analyzed by statistical software package SPSS version 20. There were used the experts' evaluation methods based on experts' surveyed sample analysis. The Kendall's coefficient of concordance (W) was selected for quantitative assessments of experts' decisions data analysis. In addition for PSAUAV technical conditions evaluation was used the hierarchical clustering.
\end{abstract}

KEY WORDS: Unmanned Aircraft Vehicle, multidimensional database, hierarchical clustering, Kendall W

\section{Introduction}

The chosen investigations have become currently relevant because the activity of unmanned aerial vehicles (drones) in the world is increasing over the last 10 years. The unmanned aerial vehicles are one of the fasters growing and most exciting technologies anywhere in the world. There are many companies that predict the UAVs market variations. These companies for market analysis used the different sources of information and methodologies, but their forecast analysis proves that the market of the UAVs is growing [1]. More than eighty countries produced the unmanned aerial vehicles for the different purposes, but there are only about twenty-five countries which producing military UAVs [2]. Some examples of commercially available LSS UAVs, a glider type UAS, commercially popular quadcopters, and a jet turbine based highvelocity UAVs are shown in the Fig. 1.

The challenges of detecting low, slow, and small (LSS) unmanned aerial vehicles (UAVs) becoming an important capability for the maintenance of security. Consumer grade LSS UAVs are becoming increasingly complex, and represent a diverse new threat which must be addressed by physical security systems of the future. The conclusion is drawn from internal discussions and external reports are the following; detection of LSS UAVs is a challenging problem that cannot be achieved with a single detection modality for all potential targets. For security purposes, the UAVs were categorized by their mass and typical capabilities that are associated with each class, as shown in Table 1[3]. There we can see that the first class UAVs includes anything less than $150 \mathrm{~kg}$, while second class extends to the larger types between 150 and 600 $\mathrm{kg}$. This upper class is, for now, generally restricted to military aircraft.

The UAVs had been used only for military purposes until a few years ago, but now the features and capabilities of technology used for military, commercial and civilian purposes UAVs becoming very close. For this reason, the UAVs have recently joined to the other common things which can become suddenly lethal weapons. Classification of LSS UAVs, especially classification in the presence of background clutter (e.g., urban environment) or other non-threating targets (e.g., birds), is under-explored. However information of accessible technologies is sparse, numerous of the current possibilities for UAS detection seem to be in their beginning when compared to more established ground-based air protection systems for larger and/or faster threats. In addition, there can be mentioned that all companies currently providing or developing technologies to combat the UAVs safety and security problem are certainly worth investigating, however, no company has provided the statistical evidence necessary to support robust detection, identification, and/or neutralization of LSS UAVs targets.

Corresponding author.

E-mail address: Svajone.Bekesiene @lka.lt 


\begin{tabular}{|c|c|c|c|c|}
\hline Class $/$ Weight & Category/ Weight & Operating Altitude & Operation Radius & Carrying Payload \\
\hline $\mathrm{I}(<150 \mathrm{~kg})$ & Micro $(<2 \mathrm{~kg})$ & to $90 \mathrm{~m}$ & $5 \mathrm{~km}$ & $0.2-0.5 \mathrm{~kg}$ \\
\hline $\mathrm{I}(<150 \mathrm{~kg})$ & Mini $(2-20 \mathrm{~kg})$ & to $900 \mathrm{~m}$ & $25 \mathrm{~km}$ & $0.5-10 \mathrm{~kg}$ \\
\hline $\mathrm{I}(<150 \mathrm{~kg})$ & Small $(<150 \mathrm{~kg})$ & to $1500 \mathrm{~m}$ & $50-100 \mathrm{~km}$ & $5-50 \mathrm{~kg}$ \\
\hline $\mathrm{II}(150-600 \mathrm{~kg})$ & Tactical & to $3000 \mathrm{~m}$ & $200 \mathrm{~km}$ & $25-200 \mathrm{~kg}$ \\
\hline
\end{tabular}

Military conflict in Ukraine has shown the growing use of such aircraft in military and civil conflicts. The UAVs have been becoming a real threat and weapon in present-day asymmetric warfare, terrorist attacks or malicious uses. Due to the capabilities and size of various drones, their shoot down has become difficult. Units of the Lithuanian Armed Forces, military exercises, the military equipment of Lithuania and its NATO allies attract more and more attention from the people. Such factors have led to the intensive use of drones over military areas and other restricted areas.

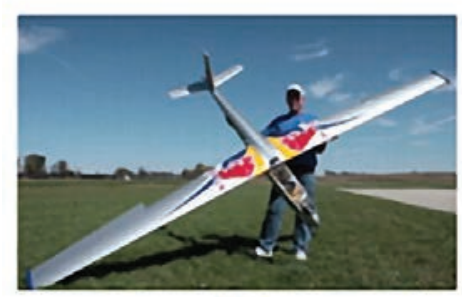

(a)

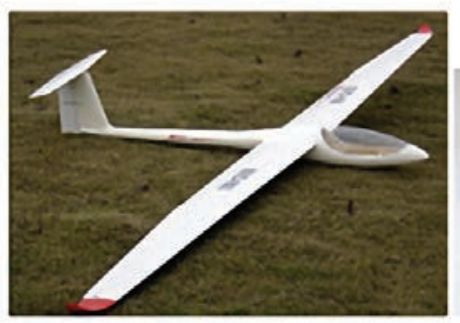

(d)

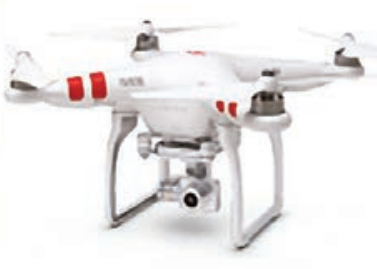

(b)

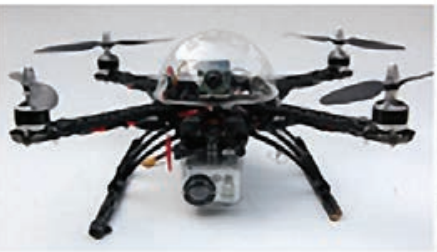

(e)

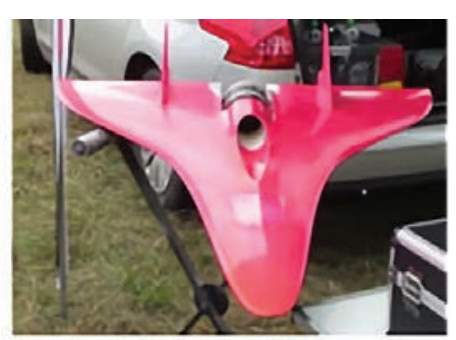

(c)

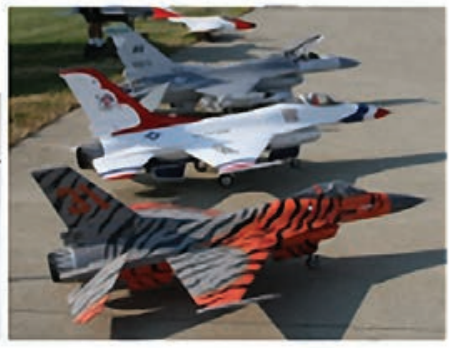

(f)

Fig. 1. Commercially available LSS UAVs (a) and (d) show two variants of a glider type UAS, (b) and (e) show commercially popular quadcopters, (c) and (f) show jet turbine based high-velocity UAVs [3]

Airborne threats coming from the drones have revealed a security loophole in the Lithuanian Armed Forces.

Ultimately, the appropriateness of the methods for qualification or neutralization of LSS UAVs targets within the wanted setting is what will order their use and/or implementation in future ground-based aerial defence systems or LSS UAVs qualification systems. Also, the use of UAVs ammunitions and missiles for security or safety protection is obviously not ideal in a heavily populated civilian environment. On the other hand, these methods may be appropriate for engaging targets on a hostile battlefield. The decision for use of such devices must be heavily influenced by the inherent risks in each (e.g. collateral damage or ineffectiveness), and whether the consequences of those risks are determined to be acceptable. However, Lithuanian Armed forces do not have any safety protection system that can effectively fight against hostile drones. This study was provided on an existing nine protection systems used against remotely controlled miniunmanned aircraft systems engineering analysis with the goal to help to make the decisions in defining a system that can be adaptive to the Lithuanian Armed Forces current and future needs.

\section{Combat Concept and Protection Systems}

The UAVs which have the possibility to perform hazardous, malicious or unwanted actions are considered threats. This includes devices intending to carry out a hostile mission, being operated by an unsafe individual, or crossing into a sensitive area. UAVs threats must be appropriately dealt with by security systems, where the type and extent of mitigation techniques depend on the situation and environment. The three steps of protection against UAVs was clarified by the Ministry of National Defence (MND). The following combat concept developed by MND was chosen as background for on how to combat the unmanned aerial vehicles (drones). The MND concept was separate into three combat modules: 
- Module I - Detection. The first there is necessary to collect some phenomenological information captured by a sensor.

- Module II - Identification / Tracking. The received data in the detection phase analysis, with the goal being to separate real targets from highly clustered, noisy background data. This step of the analysis is performed solely by a human.

- Module III - Neutralization. Once a target is positively identified in the previous step, additional action must be taken to deny mission success, including the potential for target neutralization. An overview of detection methods, drone neutralization options, specific blocked frequencies, and the main weapon - the jammer.

In order to accurately select a protection system for the Lithuanian Armed Forces (LAF), which will later be used by all LAF units and full fill security-compliant requirements. The working group under the Lithuanian Force Planning Department prepared the ORD documentation by the weightier criterion for acquiring security measures against unmanned aircraft vehicles. The analysis was done under the military purposes which are described in detail in the Operational Requirements Document (ORD). The existing nine protection systems such as Blighter Surveillance Systems/UK; Aquila Defence Group/ Switzerland; HENDSOLDT/ Germany; Bukovel/ Ukraine; Elbit Systems/ Israel; NT Service/ Lithuania; Radio Hills Technologies/ USA; MOOG/ USA; Rohde\&Schwarz/ Denmark, were analyzed. Technical specifications for acquisitions are drawn up and the detailed requirements of the measures are set out on the basis of ORD.

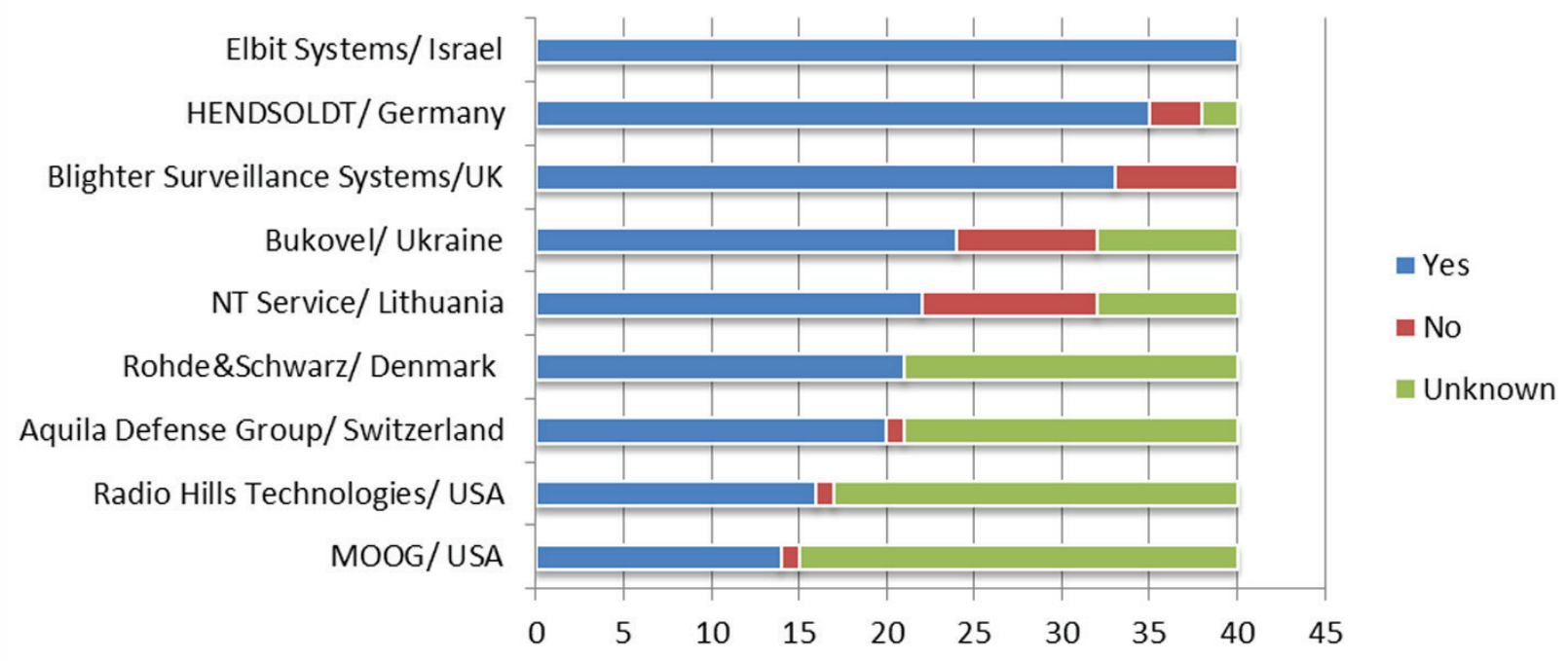

Fig. 2. The evaluation of capabilities of the each of nine systems

The technical information about nine existing protection systems (EPS) was composed after deep analyses of the ORD documentation where were jagged all requirements for the protection system which can be used in the Lithuanian Armed Forces. The nine EPS technical documentation analysis helped to disclose the characteristics and/or capabilities on the each of these systems. In addition, the nine chosen systems were statistically evaluated by the following three combat modules for unmanned aerial vehicles. All analysis was done by the separating essential functionality for each of the nine ESP with summarizing the main requirements in accordance with three principles: accuracy, reasonableness and completeness. For example, the anti-poverty task force distinguishes three key functions: detection, identification and neutralization.

According to the ORD requirements, the protection system that was searched for LAF needs had to have the jammer. It seems that the chosen system has to be able to use for tampering blocking, transmitting, blocking, or otherwise kinetically manipulating the UAVs. The ORD documentation precise requirements make it possible to choose the most appropriate protection, to determine the detailed technical requirements for military equipment and to properly understand the request for information received by enterprises. This analysis showed the Lithuanian Armed Forces needs and helped to identify the most appropriate device. In this survey, the detailed possibilities of nine EPS were compared with the respect to the minimum of main parameters (forty parameters were evaluated). The receiving result is presented in the graphical visualization in the Fig. 2. All forty criteria for each of nine EPS were evaluated as follows: if the EPS system meets the criteria under consideration - "YES", if not - "NO", in other cases where the manufacturer did not mention anything about the assessed requirement it was weighed as "Unknown". In the Fig. 2, security measures of each nine EPS are ranked according to the criteria of the "YES" criterion from the most suitable to the least. Moreover, this graphical analysis discloses the possibility to see one more tendency: the more systems meet the requirements, they have the less the unknown and unavailable system components that are specified in the ORD requirements. We can also notice another trend that if the "NO" criteria increase, the "YES" criterion decreases. The statistical analysis showed that all of the forty criteria were met by the Israeli company Elbyt Systems, the second system meeting the highest criteria is HENDOLDT with 35 requirements. The United Kingdom system remains in the third position of the chart with only 33 matching requirements.

Having analyzed the existing protective systems measures which were on interest for the Ministry of Lithuania National Defense, there were clarified their possibilities, advantages and disadvantages. Due to the fast-paced technology, 
there is no ideal system or product against UAVs. All analyzed systems and products do not have a final solution that can be effective in the future. Therefore, for Lithuanian Armed Forces there was very important that the manufacturers must provide for the possibility of continuous updating of equipment, the introduction of new components to adapt to newly emerging threats. There was clarified that the commercial equipment designed to fight against civilian aircraft is ineffective against modified and/or military aircraft. For these reasons, the research was extended with additional multidimensional data analysis. The mathematical background and the results of these analyses are presented below.

\section{Investigation Methods and Mathematical Background}

The goal of comprehensive investigations was to analyze selected nine EPS and identify groups that are similar to each other by technical features but differ from EPS parameters in other groups. It can be intellectually satisfying, profitable, or sometimes both to manage this without statistical analysis because it can't be known who or what belongs in which group and a number of groups can be known only after clustering analysis. The SPSS statistical package has three different procedures that can be used to cluster collected data [16]. From the methodological point of view, there was chosen the agglomerative hierarchical clustering analysis and similarity of groups was measured by Euclidean distance, which is suitable for only continuous variables and has a possibility to choose a statistic that quantifies how far apart (or similar) two cases (two EPS) are. Using collected technical parameters data set and agglomerative hierarchical clustering with selected a median method for forming the similar groups. In this way from nine EPS, there were realized the EPS with similar protection promises against a remotely controlled unmanned aircraft systems.

In addition, was conducted the experts' opinion evaluation analysis based on experts' surveyed sample. The Kendall's coefficient of concordance (W) was selected for experts' data assessment. The statistical software package SPSS version 20 was used for the collected experts' opinions data analysis [16].

\subsection{Mathematical Background for Agglomerative Hierarchical Clustering Analysis}

The hierarchical clustering method determines the overall structure of interconnections of all clusters and also helps to select the optimal number of clusters. The agglomerative hierarchical clustering method is divided into combining and dividing methods. All methods are assessing the proximity by objects. In this analysis, there was used the straightforward way to assess two objects' closeness by drawing a straight line between them. This type of distance is also referred to as Euclidean distance or straight-line distance and is the most commonly used type when it comes to analyzing ratio or interval-scaled data. In the mathematical point of view it can be described by the equation:

$$
\operatorname{Dist}(x, y)=\sqrt{\sum_{i=1}^{n}\left(x_{i}-y_{i}\right)^{2}},
$$

where $\mathrm{x}_{\mathrm{i}}$ and $\mathrm{y}_{\mathrm{i}}$ are the cases measurement vectors. Some analysis of clustering methods can be conducted with any kind of similarity or distance measure between cases, but there are three methods: Ward's method, Centroid method and Median method, which use squared Euclidean distances:

$$
\operatorname{Dist}(x, y)=\sum_{i=1}^{n}\left(x_{i}-y_{i}\right)^{2} \text {. }
$$

In this case, was selected a squared Euclidean distances measure of similarity or dissimilarity (equation 2) and chosen a median clustering algorithm. This method takes into consideration the size of a cluster, rather than a simple mean [16, 17]. With the median method, the two clusters being combined are weighted equally in the computation of the centroid, regardless of the number of cases in each. This allows small groups to have an equal effect on the characterization of larger clusters into which they are merged. The investigation results are presented in the fifth section below. All analysis was following the agglomerative hierarchical clustering mathematical background $[16,17]$.

\subsection{Mathematical Background for Experts’ Data Assessment}

Simulations were done by SPSS and are presented in the section 5 to compare empirically the classical $\chi^{2}$ test of the coefficient of concordance.

There are two ways to found for computing Kendall's W statistic, but they lead to the same result. S or S' is computed first from the row-marginal sums of ranks $\mathrm{R}_{\mathrm{i}}$ received by the objects:

$$
S=\sum_{i=1}^{n}\left(R_{i}-\bar{R}\right)^{2}
$$

$$
S^{\prime}=\sum_{i=1}^{n} R_{i}^{2}=S S R .
$$

$\mathrm{S}$ is a sum-of-squares statistic over the row sums of ranks $R_{i} \cdot \bar{R}$ is the mean of the $R_{i}$ values. Following that, Kendall's $\mathrm{W}$ statistic can be obtained from either of the following formulas: 


$$
W=\frac{12 S}{p^{2}\left(n^{3}-n\right)-p T}
$$

or

$$
W=\frac{12 S^{\prime}-3 p^{2} n(n+1)^{2}}{p^{2}\left(n^{3}-n\right)-p T},
$$

where $\mathrm{n}$ is the number of objects, $\mathrm{p}$ the number of judges. T is a correction factor for tied ranks (Siegel 1956, p. 234; Siegel and Castellan 1988, p. 266; Zar 1999, p. 446):

$$
T=\sum_{k=1}^{m}\left(t_{k}^{3}-t_{k}\right)
$$

in which tk is the number of tied ranks in each $(\mathrm{k})$ of $\mathrm{m}$ groups of ties. The sum is computed over all groups of ties found in all p columns (judges) of the data table.

Kendall's W statistic is an estimate of the variance of the row sums of ranks $R_{i}$ divided by the maximum possible value the variance can take; this occurs when all judges are in total agreement; hence $0 \leq \mathrm{W} \leq 1$. To derive the formulas for $\mathrm{W}$ given above, one has to know that the sum of all ranks in the data table is $p(n+1) / 2$ and that the sum of squares of all ranks is $p^{2} n(n+1)(2 n+1) / 6$. Friedman's $\chi^{2}$ statistic is obtained from W using the formula:

$$
\chi^{2}=p(n-1) W
$$

This quantity is asymptotically distributed like chi-square with (n -1) degrees of freedom. This allows us to test W for statistical significance. The results get after our investigations are discussed in the fourth section below.

\section{Experts' Data Analysis Results}

There were questioned the ten experts. The nine of the interviewed experts are officers or soldiers and only one is the civilian expert. All experts belong to the same group which was arranged for the fighting against the UAVs. Almost all experts have higher education, only one has the higher non-university degree. The six of experts' are in this team for only one year, the two are about two years and only two of them were with experience of five years. The age of questioned ten experts varied from 25 to 40 years. The detailed characteristics are known for the author, but will not be presented in this paper. All of these experts have a lot of competence and knowledge working with UAVs, but in the different field.

For this research was selected the list of sixteen technical specifications which broadly define three steps to UAVs threat. The technical criteria codes used in experts ${ }^{`}$ data statistical analysis are presented in Table 3 and the brief description can be presented like this:

- Five technical criteria for UAVs detection: CR1, CR2, CR11, CR13, CR16;

- Five technical criteria for UAVs identification / Tracking: CR3, CR4, CR12, CR15, CR14;

- Six technical criteria for UAVs neutralization: CR5, CR6, CR7, CR8, CR9, CR10.

The data collected from questioned ten experts were analyzed by SPSS package, the inferential statistic was tested and the null hypothesis of equality of concordance coefficient to zero was verified. But there was impossible to make the conclusion, that all ten experts' opinions are close because the experts' assessments describe Kendall's concordance coefficient $(\mathrm{Wa}=0,174)$ and p-value $(0,037)$. In addition, the analysis was repeated with the four experts' group. The six experts' were eliminated from the continual analysis of the reason that they work in the experts teem only one year. The surveyed four experts opinions were very similar, this was shown by Kendall's concordance coefficient, which was high enough $(\mathrm{Wa}=0,580)$ and $\mathrm{p}$-value $(0,007)$.

The continual analysis result when the preselected group of four experts according to their specific experience was used allowed us to use the expert judgment for decision making. In this way were determined the most important technical criteria, which can be clarified as the main technical parameters for existing protection systems. 
The protection system technical criteria evaluation analysis

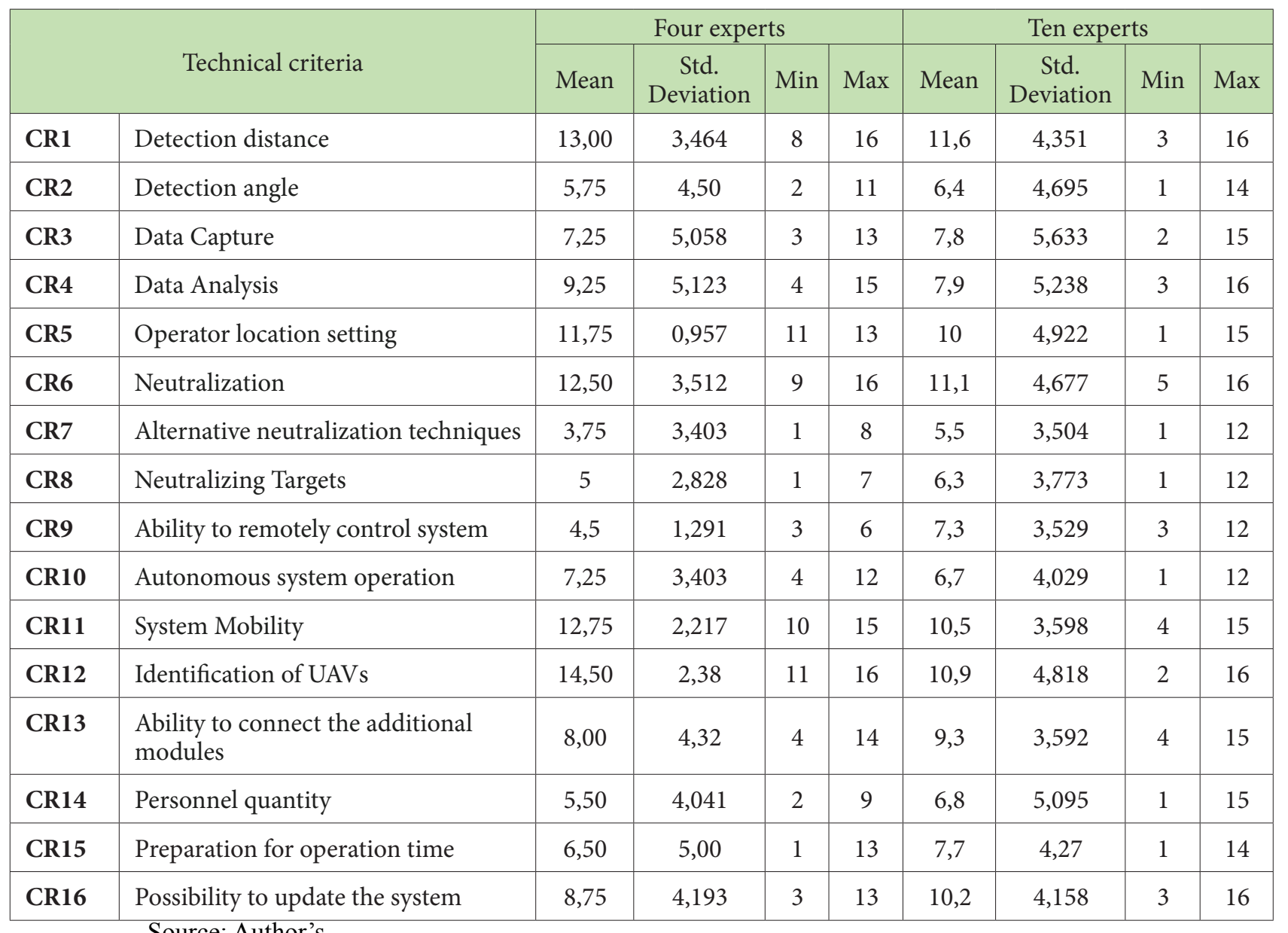

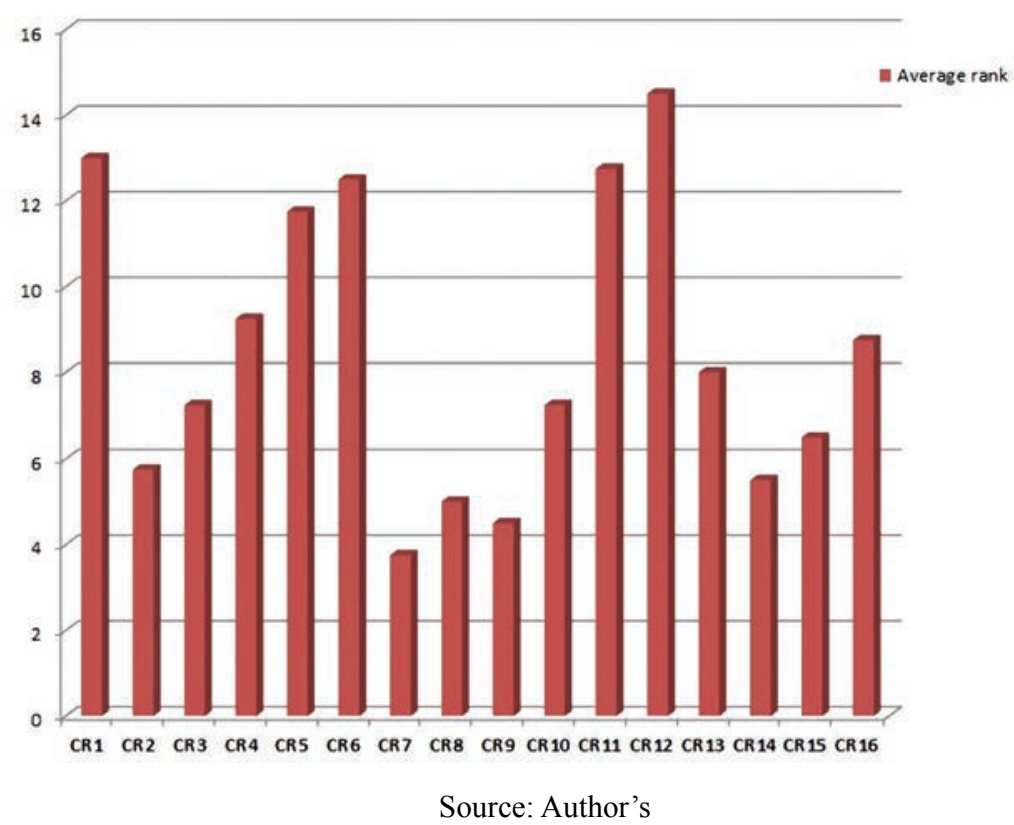

Fig. 2. Result for technical criteria ranking by experts' opinion

According to the experts' opinion five of sixteen criteria were marked as important (Fig. 2). The first important feature for existing protection systems was chosen the CR12 - Identification of UAVs (evaluated in 58 points of 64), the second in the queue was CR1 - Detection distance (evaluated in 52 points of 64), the third was CR11 - System Mobility (evaluated in 51 points of 64), the fourth was CR6 - Neutralization (evaluated in 50 points of 64) and the fifth was CR5 - Operator 
location setting (evaluated in 47 points of 64). Moreover, after this analysis by following experts' recommendations for protection system capabilities to fight against unmanned aircraft vehicles, we can conclude as the most important possibilities were selected two: the Identification of UAVs and the Detection distance.

This research continues with the additional statistical analysis, which solves in part research goals and objectives. The statistical software package SPSS version 20 was used to perform the agglomerative hierarchical clustering analysis [17]. The results are presented in the next section.

\section{Agglomerative Hierarchical Clustering Results}

As was mentioned above, the agglomerative hierarchical clustering analysis was used to group by similarity nine existing protection systems. The two of EPS were manufactured in USA: Radio Hills Technologies and MOOG. The others - mass-produced: in the Israel (Elbit Systems), in the Germany (HENDSOLDT), in the Ukraine (Bukovel), in the Switzerland (Aquila Defense Group), in the Denmark (Rohde\&Schwarz), in the Lithuania (NT Service) and one - in the UK (Blighter Surveillance Systems). All parameters for the hierarchical clustering analysis for these nine existing protection systems are well known for author, but in safety reasons aren't detail in this article.

Table 4

Dissimilarity matrix for nine EPS

\begin{tabular}{|c|c|c|c|c|c|c|c|c|c|}
\hline \multirow[b]{2}{*}{ Protection System } & \multicolumn{9}{|c|}{ Squared Euclidean Distance } \\
\hline & 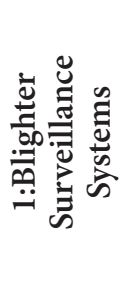 & 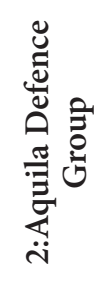 & 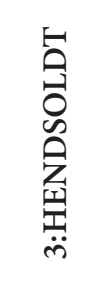 & $\begin{array}{l}\vec{D} \\
\dot{0} \\
\dot{y} \\
\dot{0} \\
\dot{q}\end{array}$ & 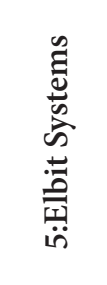 & 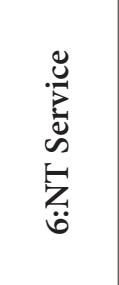 & 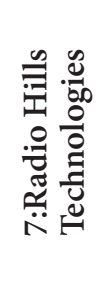 & 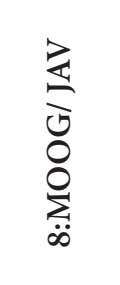 & 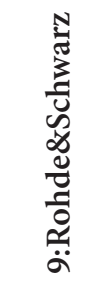 \\
\hline $\begin{array}{l}\text { 1:Blighter Surveillance } \\
\text { Systems }\end{array}$ & .000 & 4.953 & 12.959 & 20.020 & 18.112 & 7.297 & 14.105 & 16.827 & 6.593 \\
\hline 2:Aquila Defence Group & 4.953 & .000 & 19.113 & 25.389 & 14.351 & 5.557 & 12.063 & 25.139 & 4.718 \\
\hline 3:HENDSOLDT & 12.959 & 19.113 & .000 & 27.774 & 22.193 & 14.170 & 9.397 & 14.593 & 13.605 \\
\hline 4:Bukovel & 20.020 & 25.389 & 27.774 & .000 & 26.084 & 18.308 & 22.783 & 36.462 & 18.434 \\
\hline 5:Elbit Systems & 18.112 & 14.351 & 22.193 & 26.084 & .000 & 17.994 & 22.068 & 36.666 & 13.431 \\
\hline 6:NT Service & 7.297 & 5.557 & 14.170 & 18.308 & 17.994 & .000 & 4.775 & 14.194 & .894 \\
\hline 7:Radio Hills Technologies & 14.105 & 12.063 & 9.397 & 22.783 & 22.068 & 4.775 & .000 & 13.210 & 5.009 \\
\hline 8:MOOG & 16.827 & 25.139 & 14.593 & 36.462 & 36.666 & 14.194 & 13.210 & .000 & 16.764 \\
\hline 9:Rohde\&Schwarz & 6.593 & 4.718 & 13.605 & 18.434 & 13.431 & .894 & 5.009 & 16.764 & .000 \\
\hline
\end{tabular}

Source: Author's

As for Lithuania Armed Force's future plans, the parameters were specified with respect to all requirements which are enumerated by the Ministry of National Defence of Lithuania in the ORD. In this way for each protection system constructed to fight against hostile drones were collected the eight measures.

Table 5

Agglomeration Schedule

\begin{tabular}{|c|c|c|c|c|c|c|}
\hline \multirow{2}{*}{ Stage } & \multicolumn{2}{|c|}{ Cluster Combined } & \multirow{2}{*}{ Coefficients } & \multicolumn{2}{|c|}{ Stage Cluster First Appears } & \multirow{2}{*}{ Next Stage } \\
\cline { 2 - 3 } & Cluster 1 & Cluster 2 & & Cluster 1 & Cluster 2 & \\
\hline 1 & 6 & 9 & .894 & 0 & 0 & 2 \\
2 & 6 & 7 & 4.668 & 1 & 0 & 4 \\
3 & 1 & 2 & 4.953 & 0 & 0 & 4 \\
4 & 1 & 6 & 7.045 & 3 & 0 & 5 \\
5 & 1 & 3 & 10.819 & 4 & 0 & 7 \\
6 & 1 & 8 & 11.914 & 5 & 0 & 8 \\
7 & 1 & 5 & 23.185 & 6 & 0 & 0 \\
8 & 1 & 4 & 19.995 & 7 & 0 & 0 \\
\hline
\end{tabular}

Source: Author's 
The agglomerative hierarchical clustering analysis helped to identify similar groups by technical criteria parameters such as RF scanner; radar, an acoustic sensor, the thermal sensor, the electronica optical sensor, the detecting module, the identification module and neutralization module. All chosen variables in this analysis under consideration are measured on different scales and levels. There is the possibility to resolve this problem by standardizing the data prior to the analysis. There are available different standardization methods in the hierarchical clustering procedure in SPSS. The variables can be standardized in different ways. There is the possibility to compute for variables standardized scores or divide by just the standard deviation, range, mean, or maximum. This resulted in all variables contributing more equally to the distance measurement.

Cluster Membership

Table 6

\begin{tabular}{|c|c|c|c|}
\hline Case & 4 Clusters & 3 Clusters & 2 Clusters \\
\hline 1:Blighter Surveillance Systems & 1 & 1 & 1 \\
\hline 2:Aquila Defense Group & 1 & 1 & 1 \\
\hline 3:HENDSOLDT & 1 & 1 & 1 \\
\hline 4:Bukovel & 2 & 2 & 2 \\
\hline 5:Elbit Systems & 3 & 3 & 1 \\
\hline 6:NT Service & 1 & 1 & 1 \\
\hline 7:Radio Hills Technologies & 1 & 1 & 1 \\
\hline 8:MOOG & 4 & 1 & 1 \\
\hline 9:Rohde\&Schwarz & 1 & 1 & 1 \\
\hline
\end{tabular}

Source: Author's

There was chosen the simple $\mathrm{z}$ standardization for this analysis. This method rescaled each variable to have a mean of 0 and a standard deviation of 1 . The data standardization was used, by the reason to reduce or inflate the variables' influence on the clustering solution. In addition, the median algorithm was selected for clustering. In this analysis, each of clusters pair being combined was weighted equally in the computation of the centroid, regardless of the number of cases in each. One of the results get after this analysis is the dissimilarity matrix for nine EPS which is presented in Table 4.

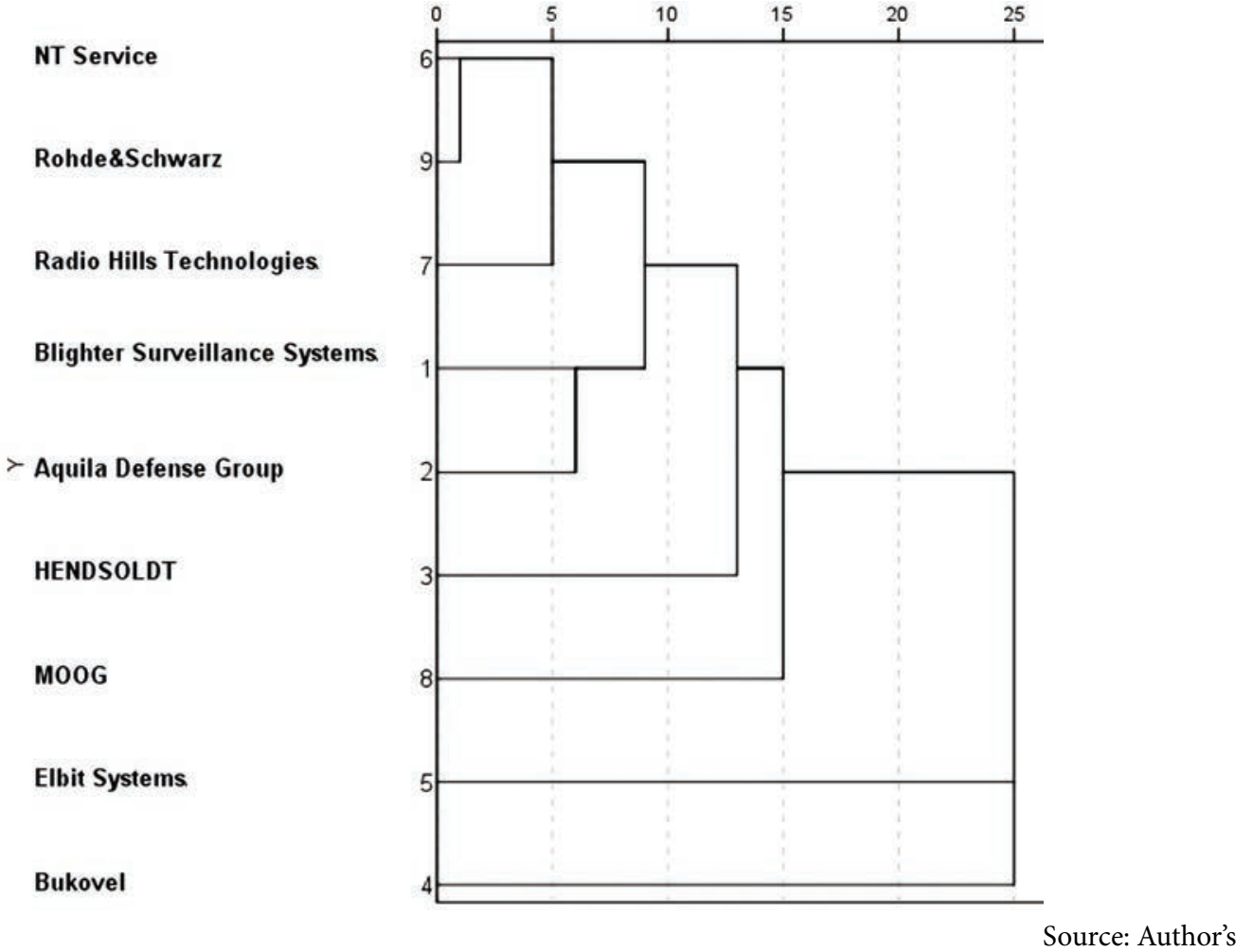

Fig. 3. The dendrogram using median linkage between groups 
To get the squared Euclidean distance between each pair of EPS, there were squared the differences in the eight scores that were assigned to each of the nine EPS pairs. There were weighing the 72 scores for each EPS. The distances get after calculations are shown in Table 4, the proximity matrix. The presented distance matrix is symmetric. All of the numbers on the diagonal are equal to zero since an EPS does not differ from itself. The smallest difference between two EPS to 0.894. This is the distance between Denmark (Rohde\&Schwarz) and Lithuania (NT Service) protection systems. The largest distance in the proximity matrix equals to 20.020 and occurs between the UK (Blighter Surveillance Systems) and the Ukraine (Bukovel) EPS.

There was used the possibility to choose the different clusters number in the agglomerative hierarchical cluster analysis. In purpose to accurately represent collected data, there were determined by this analysis two, three and four clusters which grouped by similarity different EPS. The cluster membership analysis results are presented in Table 6. There you can see how similarly constructed clusters are when was created additional clusters.

The agglomeration schedule which can help to make the decision about clusters number is presented in Table 5. The distance statistic value was used to form the cluster because for this analysis the median method was applied. The figures in the column labelled Coefficients indicate how unlike the clusters being combined are. In this research was used the dissimilarity measures, so the small coefficients indicate about fairly homogenous clusters are being attached to each other and large coefficients indicated that dissimilar clusters are combining [17].

The visual representation of the distance at which clusters are combined is shown in the dendrogram (Fig. 3). The dendrogram is read from left to right. The observed distances are rescaled to fall into the range of 1 to 25 , so you don't see the actual distances; however, the ratio of the rescaled distances within the dendrogram is the same as the ratio of the original distances. The first vertical line, corresponding to the smallest rescaled distance, is for the NT Service (Lithuania) and ROHDE \& SCHWARZ (Denmark). The second vertical line corresponds to the NT Service (Lithuania) and Radio Hills Technologies (USA). What is presented in the plot in the Fig. 3 is the graphical view of information that is accessible in the agglomeration schedule, Table 5. By this way there are possible to conclude, that in the last two steps, fairly dissimilar clusters are combined: the Elbyt Systems (Israel) and the Blighter Surveillance Systems (UK) with 23.185 distance coefficient; the Blighter Surveillance Systems (UK) and Bukovel (Ukrainian) with 19.995 distance coefficient. The Elbyt Systems (Israel) and Bukovel (Ukrainian) are the furthest away from other clusters. This is due to the fact that their technical characteristics are significantly better than the EPS in the first cluster.

Based on the obtained results we can conclude that after agglomerative hierarchical clustering analysis which was done on nine existing protection systems inattention to eight chosen technical criteria measures, four fairly different clusters were identified:

- the first cluster can be presented as Blighter Surveillance Systems (UK), Aquila Defense Group (Switzerland), HENDSOLDT (Germany), NT Service (Lithuania), Radio Hills Technologies (USA) and Rohde \& Schwarz (Denmark);

- the second cluster considers only one EPS - Bukovel (Ukrainian);

- the third cluster considers only one EPS - the Elbyt Systems (Israel);

- the fourth cluster considers only one EPS - the MOOG (USA).

The results of postponed hierarchical clustering analysis let us realize which EPS belongs in which group and additional was disclosed a number of similar groups (clusters). To manage this without hierarchical clustering analysis it can be intellectually satisfying.

\section{Conclusions}

The analysis of precise requirements listed in the ORD documentation makes it possible to choose the most appropriate protection, to determine the detailed technical requirements for military equipment and to properly understand the request for information received by enterprises. In addition, the ORD analysis showed the Lithuanian Armed Forces needs and helped to identify the most appropriate device.

The detailed capabilities survey of nine EPS with the respect to the minimum (forty parameters were evaluated) of main parameters let us clarify nine existing protective systems possibilities, advantages and disadvantages. All criteria were encountered by the protection system Elbyt Systems which is mass-produced in Israel, the second system meeting the highest criteria was HENDOLDT (Germany) with 35 requirements. The United Kingdom system Blighter Surveillance Systems remains in the third chart position with 33 matching requirements. Moreover, there was realized that all analyzed existing protection systems and products had the weakness. Also was realized that no one of nine EPS does not have a final solution that can be effectively used in the future.

The research on existing protection systems shows us that due to the fast-paced technology, there is no ideal system or product against UVAs. The manufacturers didn't provide for the possibility of continuous updating of equipment, installing new components to adapt to newly emerging threats. Also can be mention, that the commercial equipment designed to fight against civilian aircraft is ineffective against modified and/or military aircraft.

With the assistance of a specific experience group of experts were marked as important only five of sixteen criteria: the CR12 - Identification of UAVs (evaluated in 58 points of 64), the CR1 - Detection distance (evaluated in 52 points of 64), the CR11 - System Mobility (evaluated in 51 points of 64), the CR6 - Neutralization (evaluated in 50 points of 64) and the CR5 - Operator location setting (evaluated in 47 points of 64).

The agglomerative hierarchical clustering analysis results showed the closest and the remote protection systems used for aircraft unnamed vehicles which can be pointed for Lithuanian Armed Forces demands. In purpose to accurately represent collected data, there were determined by this analysis two, three and four clusters which were grouped by different EPSs' similarity. The precise classification was met only in the four cluster group. 


\section{References}

1. Nagpal K. Unmanned aerial vehicles (UAV) market. Defence ProAc, New Delhi, Delhi, India, 2012.

2. Military Factory. Unmanned Aerial Vehicles (UAVs), Unmanned Combat Aerial Vehicles (UAVs) and Drone Aircraft. U.S., 2016.

3. Birch G.C., Griffin J. C., Erdman M. K. UAS Detection, Classification, and Neutralization: Market Survey 2015. Issued by Sandia National Laboratories, USA, 2015, pp.74. Available from:

4. http://prod.sandia.gov/techlib/access-control.cgi/2015/156365.pdf

5. Williams K. W. A summary of Unmanned Aircraft accident/incident data: Human factors implications. Technical Report No. DOT/FAA/AM-04/24, Washington, DC. U.S. Department of Transportation, Federal Aviation Administration, Office of Aerospace Medicine. 2004.

6. Ryan J. Wallace, Jon M. Loffi. International journal of aviation, aeronautics, and aerospace „Examining Unmanned Aerial System Threats \&Defenses: A Conceptual Analysis", 2015, p. 6-13.

7. Otto R.P. Air Force ISR 2023: Delivering Decision Advantage; Headquarters the United States Air Force:

8. Washington, DC, USA, 2013.

9. Dempsey M. E. Intelligence, Surveillance, and Reconnaissance Joint Force 2020 White Paper; U.S. Army:

10. Washington, DC, USA, 2014.

11. Cluster Analysis Basic Concepts and Algorithms [online cit.: 2017-07-22]. Available from:

12. https://www-users.cs.umn.edu/ kumar/dmbook/ch8.pdf

13. Bekešienė S., Guščinskienė J., Dvilaitis G. Intelligent Applications in the Development of Death Prevention by Suicide while on Active Duty, Active and Reserve Components. Kaunas: Technologija, 2015, p. 56-65. ISSN 23450088 (Print).

14. Seffers, G. Joint Aerial Layer Network Vision Moves Toward Reality. [online cit.: 2017-06-22].

15. Available from: http://www.afcea.org/content/?q=node/11123

16. Schechter, E. UAV s Could be Next Step for Electronic Warfare [online cit.: 2016-06-22].

17. Available from: http://archive.c4isrnet.com/article/20140507/C4ISRNET08/305070006/UAVs-could-next-stepelectronic-warfare

18. Cevik, P.; Kocaman, I.; Akgul, A.; Akca, B. The Small and Silent Force Multiplier: A Swarm UAV-Electronic Attack. J. Intell. Robot. Syst. 2013, 70, 595-608.

19. Callam, A. DroneWars: Armed Unmanned Aerial Vehicles. Int. Aff. Rev. 2010, 18, 3.

20. Lockheed/Piasecki Team Tackles Cargo UAV. 2014. [online cit.: 2016-07-22]. Available from: http://aviationweek. com/awin/lockheedpiasecki-team-tackles-cargo-uav

21. Myers M. New funds to aid coast guard in adopting a UAV. Navy Times, 25 April 2015.

22. IBM SPSS Statistics [online cit.: 2017-07-22]. Available from:

23. http://www.ibm.com/analytics/us/en/technology/spss/

24. Xu R, Wunsch D. Survey of clustering algorithms. IEEE Trans Neural Netw 2005, 16, pp.645-678.

25. Valvanis, K.; Vachtsevanos, G. Future of Unmanned Aviation. In Handbook of Unmanned Aerial Vehicles Springer: Dordrecht, The Netherlands, 2015; pp. 2993-3009. 